RESEARCH ARTICLE

\title{
The Effect of an Ultradistance Foot Race on Thyroid, Stress Hormone Levels and the Immune System
}

\section{Dimitra Pappa ${ }^{1 *}$, Maria G Pavlatou2, Christina Kanaka-Gantenbein ${ }^{1,3}$, Flora Bacopoulou ${ }^{4}$, Christina Darviri $^{1}$, Katerina Skenderi ${ }^{5}$, Maria Tsironi ${ }^{6}$, George P Chrousos ${ }^{1,3,4}$ and loannis Papassotiriou ${ }^{7}$}

${ }^{1}$ School of Medicine, National and Kapodistrian University of Athens, Greece

${ }^{2}$ Department of Endocrinology, Diabetes and Metabolism, "Athens Medical Center", Greece

${ }^{3}$ Division of Endocrinology, Metabolism and Diabetes, First Department of Pediatrics, National and Kapodistrian University of Athens Medical School, "Aghia Sophia" Children's Hospital, Greece

${ }^{4}$ Medical School of the National and Kapodistrian University of Athens, Greece

5Laboratory of Nutrition and Clinical Dietetics, "Harokopio" University, Greece

${ }^{6}$ Nursing Department, University of Peloponnese, Greece

'Department of Clinical Biochemistry, "Aghia Sophia" Children's Hospital, Greece

*Corresponding author: Dimitra Pappa, Postgraduate Course on the Science of Stress and Health Promotion, School of Medicine, National and Kapodistrian University of Athens, Athens, Greece

\begin{abstract}
Background: The aim of the present research is to investigate the effect of an ultra-marathon race on the levels of thyroid, immune and stress system hormones. Moreover, to explore a potential correlation of the above-mentioned hormones with stress hormones.

Methodology: 30 out of the 40 participants who took part in the race, finished the $246 \mathrm{~km}$ in less than 36 hours, and were included in the study. There were 3 different sampling times: prior, after and 48 hours after the race. Blood samples were taken to identify stress and inflammation markers, such as CRP, IL-6 NTproBNP, MBL, troponin, leptin, cortisole and thyroid hormones.

Results: CRP, IL-6, SAA, NTproBNP and cortisol showed a dramatic increase after the race, much higher than their initial values, but returned to their initial equilibrium after 48 hours, except SAA which increased further. The behavior of mannose binding lectin with a similar growth trend was similar. Small fluctuations in all the thyroid hormones were observed with initially an increase in the levels, then a downward trend, reaching the initial values again. The only case where the concentration decreased after the race is that of leptin, while troponin concentrations were not detectable.
\end{abstract}

Conclusions: An ultra marathon can be a very stressful event for the body, often elevating hormone levels beyond their normal rate. A significant correlation was observed in the way cortisol changes with CRP, IL-6 and NTroBNP, which reveals the close relationship of the endocrineimmune system in extreme cases of exercise such as that of Spartathlon.

\section{Keywords}

Exercise, Marathon, Hormones, Thyroid, Immune system, Oxidative stress, Overtraining syndrome, Stress

\section{Introduction}

Spartathlon is one of the most difficult and longest running races in the world. It takes place every year starting from the Acropolis, usually every last Friday of September at 7 in the morning and ends in Sparta in front of the statue of Leonidas at 19:00 of the next day, which is the maximum time limit for the race to be completed ( $<36$ hours). Athletes run on muddy roads and climb on mountains of 1,200 meters altitude without being allowed to rest or sleep, even at night. It is a super-marathon with a fixed distance of $246 \mathrm{~km}$ where athletes compete for the best possible time. Various studies that measured biological indicators after the end of the race suggested what one would

Citation: Pappa D, Pavlatou MG, Gantenbein CK, Bacopoulou F, Darviri C, et al. (2021) The Effect of an Ultradistance Foot Race on Thyroid, Stress Hormone Levels and the Immune System. Int J Sports Exerc Med 7:207. doi.org/10.23937/2469-5718/1510207

Accepted: December 04, 2021; Published: December 06, 2021

Copyright: (c) 2021 Pappa D, et al. This is an open-access article distributed under the terms of the Creative Commons Attribution License, which permits unrestricted use, distribution, and reproduction in any medium, provided the original author and source are credited. 
logically assume, that the race causes a prolonged and very intense overexertion and overwork of the body at all levels, physical and mental [1].

Researchers are demonstrating an increasing interest in the former as they want to further explore how the human body reacts in the face of an extremely stressful situation, as is to carry out a marathon race e.g. Spartathlon. In addition, the "exercise and stress" standard can easily be used in research protocols and allows the study of the interactions of 3 systems of the human body simultaneously: the nervous, the immune and the endocrine system.

Overtraining can cause ischemic hyperemia, tissue damage, acidosis and oxidation caused by catecholamines, resulting in reduced performance and severe muscle fatigue. Many of these are the main symptoms of the overtraining syndrome. From the literature it is clear that overtraining is associated with hypothalamic-pituitary dysfunction. According to Selye's (1956), the body responds to what is perceived as a threat of homeostasis and adapts accordingly. The human body is made so that any stimulus (stressor) regardless of source and nature (environmental stressors such as rain, psychological stressors, e.g. loss, or in the case of this research, exercise) can automatically activate the autonomic nervous system to deal with it [2]. The autonomic nervous system in turn commands the neuroendocrine system and more specifically the hypothalamic-pituitary-adrenal axis (HPA axis) for its activation and cortisol production. Although the body's response to the stimulus will be the same, the nature and duration of stress can change the sensitivity of the cortisol's feedback mechanism for production volume, concentration, and duration of production [3]. The role of $\mathrm{CRH}$ is also to regulate the activity of the sympathetic nervous system and in part its behavior during exercise. It is produced by neurons in the microcellular part of the Para Ventricular Nucleus (PVN) in the anterior pituitary gland and the way it stimulates the release of ACTH is to induce the transcription of the proopiomelanocortin gene (POMC, proopiomelanocortin). The activity of the HPA axis as a result of the whole above biochemical process can be inhibited by the endogenous opioid peptide, $\beta$-endorphin, produced both at rest and during exercise [4].

The intensity, the duration as well as the type of the activity can have a huge impact on the reaction of the stress system and therefore also on the immune and endocrine system of the human body.

\section{Methods}

\section{Procedure}

Three blood samplings were performed, before, after and 48 after the end of the race. The duration of the race in order to consider the participation of the athletes valid has to be less than 36 hours. After the collection of all the samples, the analysis of the blood samples was carried out in the Biochemical Department, GNP Athens "The Hagia Sophia". The race took place with a daily temperature ranging from $5^{\circ} \mathrm{C}$ to $36^{\circ} \mathrm{C}$ and relative humidity of $60-85 \%$.

The study protocol was approved from the Bioethics Committee of the Harokopio University, Laboratory of Nutrition and Clinical Dietetics. All experimental procedures conformed to the National Health and Medical Research Council guidelines for experimentation with human subjects.

\section{Sampling and analysis}

In each blood draw, $10 \mathrm{ml}$ of blood was taken from the participants from the vein while they were sitting. Samples were taken at 3 different times, before (pre), immediately after (within 15 minutes) and 48 hours later during the post recovery period in exactly the same way. Venous blood samples were taken with sterile plastic syringes and immediately transferred to tubes containing the appropriate anticoagulant (citrate, EDTA, heparin). A quantity of plasma and serum was collected after centrifugation at $1500 \mathrm{~g}$ at $4{ }^{\circ} \mathrm{C}$ for 10 min and stored frozen at $-80{ }^{\circ} \mathrm{C}$ for maintenance until analysis.

Serum levels of the hormones T3, T4, FT3, FT4, TSH, cortisol, as well as the cardiac markers D-proBNP and Troponin-T were measured using enhanced electro ChemiLuminescence, with the immunological analyzer Roche Cobas e 411 (Roche Diagnostics Mannheim, Germany). The serum Amyloid A (SAA) and C-Reactive Protein (CRP) inflammation proteins were measured by immunofluorescence method with BN Prospec nephelometer (Siemens Siemens Healthineers, Liederbach, Germany) Leptin, Interleukin-6 (IL-6) and Mannose-Binding Lectin (MBL) concentrations were performed by immunoenzymatic methods (R\&D Systems USA, Minneapolis).

\section{Statistical analyses}

In order to investigate the primary objectives of the study, the analysis of repeated measures ANOVA was conducted. To examine the correlation between the quantitative variables of the study, an analysis was performed using the Pearson correlation coefficient, in case the normality criteria based on asymmetry and curvature was met, while in the cases of the analysis was violated, it was carried out through the Spearman correlation coefficient. The statistical analysis of the data was performed through SPSS, vol.22 for Windows (SPSS INC., Chicago, IL). The significance score was set at 0.05 for all analyzes.

\section{Results}

Table 1 summarizes the main results of the study. All the levels before the race where between the normal range of any healthy human being. Right after the end 
Table 1: Indicator levels pre, post and 48 hours the race.

\begin{tabular}{|l|l|l|l|}
\hline \multicolumn{2}{|c|}{ pre } & \multicolumn{1}{c|}{ Post } & 48h-post \\
\hline Inflammation & $3.2 \pm 0.56$ & $340.8 \pm 62.2$ & $444.6 \pm 77.3$ \\
\hline SAA (mg/L) & $0.65 \pm 0.2$ & $97.3 \pm 17.4$ & $63.8 \pm 12.1$ \\
\hline CRP (mg/L) & $0.9 \pm 0.1$ & $7781.0 \pm 2017.0$ & $0.7 \pm 0.1$ \\
\hline IL-6 (ng/L) & & $3.4 \pm 0.5$ & $2.0 \pm 0.2$ \\
\hline Thyroid Function & $1.9 \pm 0.2$ & $106.8 \pm 3.8$ & $97.6 \pm 2.9$ \\
\hline TSH (mU/L) & $98.4 \pm 3.8$ & $20.6 \pm 0.6$ & $18.3 \pm 0.4$ \\
\hline T4 (nmol/L) & $17.3 \pm 0.4$ & $1.7 \pm 0.1$ & $1.4 \pm 0.0$ \\
\hline FT4 (pmol/L) & $1.5 \pm 0.1$ & $5.4 \pm 0.3$ & $4.5 \pm 0.1$ \\
\hline T3 (nmol/L) & $4.8 \pm 0.1$ & & $2.00 \pm 0.16$ \\
\hline FT3 (pmol/L) & & $1.88 \pm 0.11$ & $0.86 \pm 0.44$ \\
\hline Immune Function & $1.63 \pm 0.13$ & $0.19 \pm 0.03$ & $12.2 \pm 5.6$ \\
\hline MBL (mg/L) & $1.41 \pm 0.14$ & & $82.1 \pm 12.1$ \\
\hline Leptin (g/L) & & $31.1 \pm 9.9$ & Non detected \\
\hline Stress & $13.9 \pm 4.3$ & & Non detected \\
\hline Cortisol (nmol/L) & & $964.6 \pm 153.9$ & \\
\hline Cardiac Stress & Non detected & & \\
\hline NT pro-BNP (ng/L) & & & \\
\hline Troponin T (g/L) & & & \\
\hline
\end{tabular}

of the race the levels of SAA, CRP and IL-6 changed dramatically with an upward trend, reaching levels hundreds of times higher than the initial ones. However, MBL increased slightly, but remained at normal levels, while leptin decreased from $1.41 \mathrm{mg} / \mathrm{ml}$ to $0.19 \mathrm{mg} /$ $\mathrm{ml}$, which is quite high to the point where the value is much lower than the normal limit. Thyroid hormone levels have all been on the rise, but still remain normal. The biggest changes were observed in cortisol levels, whose level tripled after the race from the original price as the increase in cardiac stress and more specifically NTproBNP was extreme, where levels reached the number of $964.6 \pm 153.9 \mathrm{pg} / \mathrm{ml}$ with a limit in healthy people $<120 \mathrm{pg} / \mathrm{ml}$.

After 48 hours, SAA increased even more, reaching a value of $444.6 \pm 77.3 \mathrm{mg} / \mathrm{L}$. However, the CRP decreased significantly, but remained at high levels, as did the SAA to a point much higher than the maximum normal range and much higher than the original measurement. The IL6 , despite its huge increase immediately after the race, returned to completely normal levels at the $3^{\text {rd }}$ time. The MBL rose slightly from the previous measurement, still staying within normal values. Similar was the behavior of leptin, which increased significantly from the previous measurement and tends to approach its original value before the race. There was a slight difference in thyroid hormone levels, with a slight decrease and a tendency to return to the original measurement values. The course of cortisol was also decisive, which after tripled in the measurement immediately after the race, decreased 48 hours later but not statistically significant. The drop in NT pro BNP was also large, but it remained exactly double the price compared to the measurement of the first moment. Finally, troponin concentrations at all 3 times were below the lowest detectable values and therefore undetectable.

\section{Discussion}

The immune system's reactions to exercise depends on the nature, extent, and duration of physical activity and directly depends on the ability of leukocytes to migrate through the blood to neighboring tissues $[5,6]$. Prolonged intense exercise causes systemic inflammatory changes and possible organ damage, as indicated by the dramatic increase in IL-6, CRP, SAA, and other indicators of endothelial dysfunction and specific muscle damage enzymes as reported in the Goussetis study, 2009 [7]. However, it is one of the best models that could be used in research because it offers the opportunity to study the physiological mechanisms (regardless of whether it is followed by successful repair of the damage) as any change or damage that may occur in the body at any level is reversible [8-10]. In order to be able to maintain its homeostasis intact, the body treats this condition by making a huge defense effort $[3,11,12]$. Especially in the category of professional athletes, where training programs are very intense and inconsistent with small recovery times, there is rhabdomyolysis and increased oxidative stress, factors confirming the severe inflammation in which the athlete's body is located [13-15], a fact which also justifies the extreme values observed in the second phase of the study, immediately after the end of the race.

The most significant changes in this study were observed in inflammatory hormones with a huge increase in levels after the race but also their stay at 
high levels and after 48 hours of recovery especially SAA which increased even more in the $3^{\text {rd }}$ time. The fluctuations in thyroid hormones were relatively small. Particularly increasing was the course of NTproBNP, where levels after the race reached or far exceeded normal human limits.

A similar course but of lesser intensity was the increase in cortisol, which tripled at the $2^{\text {nd }}$ phase but returned to lower values than the initial measurement after the recovery window. The leptin course was reversed where the levels reached a drop of $80 \%$ while remaining much lower than the initial measurement after 48 hours. Interesting are the relationships 48 hours before the end of the race between the thyroid hormones FT3 and FT4 and $\mathrm{MBL}$, suggesting a possible close interaction between the immune system and the endocrine system. Changes in MBL concentration have previously been associated with thyroid function in Graves' disease, where elevated $\mathrm{MBL}$ levels have affected the concentration of thyroid hormones themselves [16].

In our study, the increasing course of MBL values at all three point in time shows a steady increase caused by exercise, which can make MBL an indirect indicator of muscle tissue damage and inflammation after exercise. Leptin is a major endocrine mediator with multiple roles in various endocrine pathways, including thyroid function $[17,18]$. In this study, leptin levels immediately after the race were associated with TSH 48 hours before the race, further enhancing the case).

Margelis A and co (2005) in a similar study reports that IL-6, CRP, SAA and free plasma DNA levels increased significantly (by 8000-, 152- 108- and 10 times, respectively) from the initial reference values, in end of the race [19]. However, IL-6 levels returned to normal for 48 hours, while CRP protein, SAA A and free plasma DNA remained elevated. An indisputable consequence of a marathon is the cellular damage to which athletes are subjected [20-22]. More specifically, myocardial damage has been assessed through imaging techniques such as cardiovascular magnetic resonance imaging and ultrasound, as well as serum markers such as troponin and NT pro-BNP $[13,23]$. Heart damage is due to inflammation of the myocardium and heart fatigue caused by exercise and not necrosis, while reports of abdominal function are rather contradictory [24].

Determination of the concentration of acute phase proteins and especially those metabolized very rapidly, such as CRP and SAA, can confirm or rule out inflammation or tissue necrosis in emergency differential diagnosis $[25,26]$. Prices after the second blood draw of CRP, SAA and IL- 6 confirm the acute inflammation in which the body is located immediately after the race as they are a hundred times higher than the initial ones. C-reactive protein (CRP) as well as other acute phase proteins such as SAA, fibrinogen and MBL are produced by hepatocytes in response to the secretion of cytokines or other mediators in various conditions (including acute inflammation) or cells, but also in autoimmune or malignant diseases [27-29]. Interleukin-6, which belongs to the pro-inflammatory cytokines, is considered a potent stimulant of $\mathrm{CRH}$, resulting in hyperactivity on the HPA axis [30-33]. This hyperactivity results in increased production of ACTH and cortisol, as evidenced by the comparison of values in $2^{\text {nd }}$ and $3^{\text {rd }}$ blood sampling.

The acute inflammatory response leading to cytokine production is often involved in the pathogenesis of type $2 \mathrm{SD}$. Several studies in recently diagnosed patients with type 2 SD indicate that inflammatory markers, such as CRP and interleukin-6, are quite high [34,35]. Cortisol is also involved in the above process, which at high levels, weakens the management of glucose and makes it impossible for the body to react to insulin [36]. And in the case of thyroid in people with type 2 diabetes, high levels of cortisol in the body, prevent the conversion of inactive thyroxine (T4) to the active hormone triiodothyronine (T3), which must travel throughout the body and activate each cell of the organism [3739]. During stressful situations, the conversion of $\mathrm{T} 4$ hormone to reverse T3 (RT3 or Reverse T3) increases, which is an inactive type of $\mathrm{T} 3$ and competes with cell receptors with active $\mathrm{T} 3$, preventing it from reaching the first cells.

\section{Conclusions}

Numerous studies in marathon runners confirm that intense and prolonged exercise causes debilitating rhabdomyolysis, which remains asymptomatic and is often unrelated to the clinical symptoms often seen in these athletes. However, it is characterized as the only model for the study of acute inflammation because any damage that can be caused is reversible. As far as we know, no study has looked at muscle and not just exercise-induced damage combining so many different biomarkers like this. As well as very few studies are concerned with the overall stress of the body and not just with oxidant.

This study confirms once again that types of exercise such as the Spartathlon marathon lead the body to extreme biological states. A similarity was observed in cortisol behavior with CRP, IL- 6 and NT-proBNP as there was a dramatic increase in the above values immediately after the end of the race and their almost complete return to normal after the 48-hour recovery window, a fact which enhances the interaction of the systems of the human body and especially in our case of the endocrine with the immune system.

\section{Acknowledgements}

Funding was received from Athens University Medical School. The funding source played no role in the study design; in the collection, analysis, and interpretation of data; in the writing of the report; or in the decision to submit the report for publication. 


\section{Conflicts of Interest}

The authors have no conflicts of interest to declare.

\section{Ethical Statement}

The study protocol was approved from the Bioethics Committee of the Harokopio University, Laboratory of Nutrition and Clinical Dietetics. All experimental procedures conformed to the National Health and Medical Research Council guidelines for experimentation with human subjects.

\section{References}

1. Knechtle B, Nikolaidis PT (2018) Physiology and Pathophysiology in Ultra-Marathon Running. Front Physiol 9: 634 .

2. Stranahan AM, Lee K, Mattson MP (2008) Central Mechanisms of HPA axis Regulation by Voluntary Exercise. Neuromolecular Med 10: 118-127.

3. Luger A, Deuster PA, Kyle SB, Gallucci WT, Montgomery LC, et al. (1987) Acute hypothalamic-pituitary-adrenal responses to the stress of treadmill exercise. $\mathrm{N}$ Engl J Med 316: 1309-1315.

4. Chrousos GP (2009) Stress and disorders of the stress system. Nat Rev Endocrinol 5: 374-381.

5. Michailidis I (2012) Exercise muscle injury and oxidative stress. Thesis. Departments of Physical Education and Sports Science of the Democritus University of Thrace and the University of Thessaly.

6. Skenderi K, Tsironi M, Lazaropoulou C, Anastasiou A Matalas AL, et al. (2008) Changes in free radical generation and antioxidant capacity during ultramarathon foot race. Eur J Clin Invest 38: 159-165.

7. Goussetis E, Tsironi M, Spiropoulos A, Skenderi K, Margeli A, et al. (2009) Spartathlon, a 246-kilometer foot race: Effects of acute inflammation induced by prolonged exercise on circulating progenitor reparative cells. Blood Cells, Molecules, and Diseases 42: 294-299.

8. Sorensen B, Jones JF, Vernon SD, Rajeevan MS (2009) Transcriptional control of complement activation in an exercise model of chronic fatigue syndrome. Mol Med 15: 34-42.

9. Franchimont D, Kino T, Galon J, Meduri GU, Chrousos GP (2003) Glucocorticoids and inflammation revisited. $\mathrm{NiH}$ Clinical staff Conference. Neuroimmunomodulation 10: 247-260.

10. Ceciliani F, Giordano A, Spagnolo V (2002) The systemic reaction during inflammation: The acute-phase proteins. Protein Pept Lett 9: 211-223.

11. Singh A, Papanicolaou DA, Lawrence LL, Howell EA, Chrousos GP, et al. (1999) Neuroendocrine responses to exhaustive running in women. Med Sci Sports Exerc 31 536-542.

12. Chrousos GP (2000) Stress, chronic inflammation, and emotional and physical well-being: concurrent effects and chronic sequelae. J Allergy Clin Immunol 106: 275-291.

13. Da Ponte A, Giovanelli N, Antonutto G, Nigris D, Curcio F, et al. (2018) Changes in cardiac and muscle biomarkers following an uphill-only marathon. Res Sports Med 26: 100111.

14. Piacentini MF, Minganti C, Ferragina A, Ammendolia A,
Capranica L, et al. (2015) Stress related changes during a half marathon in master endurance athletes. J Sports Med Phys Fitness 55: 329-336.

15. Niemelä $M$, Kangastupa $P$, Niemelä $O$, Bloigu $R$, Juvonen T (2016) Acute Changes in Inflammatory Biomarker Levels in Recreational Runners Participating in a Marathon or HalfMarathon. Sports Med Open 2: 21.

16. Wahrenberg $H$, Wennlund A, Hoffstedt J (2002) Increased adipose tissue secretion of interleukin-6, but not of leptin, plasminogen activator inhibitor-1 or tumour necrosis factor alpha, in Graves' hyperthyroidism. Eur J Endocrinol 146: 607-611.

17. La Cava A, Matarese G (2004) The weight of leptin in immunity. Nat Rev Immunol 4: 371-379.

18. Perseghin G, Lattuada G, Ragogna F, Alberti G, La Torre A, et al. (2009) Free leptin index and thyroid function in male highly trained athletes. Eur J Endocrinol 161: 871-876.

19. Margeli A, Skenderi K, Tsironi M, Hantzi E, Matalas AL, et al. (2005) Dramatic Elevations of Interleukin-6 and Acute-Phase Reactants in Athletes Participating in the Ultradistance Foot Race Spartathlon: Severe Systemic Inflammation and Lipid and Lipoprotein Changes in Protracted Exercise. J Clin Endocrinol Metab 90: 3914-3918.

20. Ostergård T, Nyholm B, Hansen TK, Rasmussen LM, Ingerslev J, et al. (2006) Endothelial function and biochemical vascular markers in first-degree relatives of type 2 diabetic patients: the effect of exercise training. Metabolism 55: 1508-1515.

21. Vidotto C, Tschan $\mathrm{H}$, Atamaniuk J, Pokan R, Bachl N, et al. (2005) Responses of $\mathrm{N}$-terminal pro-brain natriuretic peptide (NT-proBNP) and cardiac troponin I (cTnl) to competitive endurance exercise in recreational athletes. Int J Sports Med 26: 645-650.

22. Deuschle M, Gotthardt U, Schweiger U, Dettling M, Holsboer F, et al. (2017) Hypothalamic-Pituitary- Adrenocortical Dysfunction in Elderly, Male Marathon Runners: Feedback Sensitivity, Stress Response, and Effects on Verbal Memory. Neuroendocrinology 105: 150-156.

23. Yoon JH, Park Y, Ahn J, Shin KA, Kim YJ (2016) Changes in the markers of cardiac damage in men following longdistance and ultra-long-distance running races. J Sports Med Phys Fitness 56: 295-301.

24. George KP, Warburton DE, Oxborough D, Scott JM, Esch BT, et al. (2011) Upper limits of physiological cardiac adaptation in ultramarathon runners. J Am Coll Cardiol 57: 754-755.

25. Kim Y, Lee S, Kim S, Shim S, Han S, et al. (2008) Differences in cytokines between non-suicidal patients and suicidal patients in major depression. Prog Neuropsychopharmacol Biol Psychiatry 32: 356-361.

26. Bachi AL, Rios FJ, Vaisberg PH, Martins M, de Sá MC, et al. (2015) Neuro-immuno-endocrine modulation in marathon runners. Neuroimmunomodulation 22: 196-202.

27. Libby $P$, Ridker $P$, Maseri A (2002) Inflammation and atherosclerosis. Circulation 105: 1135-1143.

28. Pickup JC (2004) Inflammation and activated innate immunity in the pathogenesis of type 2 diabetes. Diabetes Care 27: 813-823.

29. Temelkova-Kurkschtiev T, Henkel E, Koheler C, Karrel K, Hanefield M (2002) Sub-clinical inflammation in newly detected type II diabetes and impaired glucose tolerance. Diabetologia 45: 151. 
30. Magrini D, Khodaee M, San-Millan I, Hew-Butler T, Provance AJ (2017) Serum creatine kinase elevations in ultramarathon runners at high altitude. Phys. Sportsmed 45: 129-133.

31. Chroussos GP (1995) The hypothalamic-pituitary-adrenal axis and immune-mediated inflammation. $\mathrm{N}$ Engl $\mathrm{J}$ Med 332: 1351-1362.

32. Papanicolaou DA, Petrides JS, Tsigos C, Bina S, Kalogeras KT, et al. (1996) Exercise stimulates interleukin-6 secretion: inhibition by glucocorticoids and correlation with catecholamines. Am J Physiol 271: 601-605.

33. Tsigos C, Papanicolaou DA, Defensor R, Mitsiades CS, Kyrou I, et al. (1997) Dose effects of recombinant human interleukin- 6 on pituitary hormone secretion and energy expenditure. Neuroendocrinology 66: 54-62.

34. Didier S, Vauthier JC, Gambier N, Renaud P, Chenue B, et al. (2017) Substance use and misuse in a mountain ultramarathon: new insight into ultrarunners population? Res Sports Med 25: 244-251.
35. Tauler $\mathrm{P}$, Martinez $\mathrm{S}$, Moreno $\mathrm{C}$, Martínez $\mathrm{P}$, Aguilo $\mathrm{A}$ (2014) Changes in salivary hormones, immunoglobulin $\mathrm{A}$, and $\mathrm{C}$-reactive protein in response to ultra-endurance exercises. Appl Physiol Nutr Metab 39: 560-565.

36. Gill SK, Teixeira AM, Rosado F, Hankey J, Wright A, et al. (2014) The impact of a 24-h ultra-marathon on salivary antimicrobial protein responses. Int J Sports Med 35: 966971.

37. Neto AM, Parisi MCR, Alegre SM, Pavin EJ, Tambascia MA, et al. (2015) Relation of thyroid hormone abnormalities with subclinical inflammatory activity in patients with type 1 and type 2 diabetes mellitus. Endocrine 51: 64-71.

38. Riis AL, Hansen TK, Thiel S, Gravholt CH, Gjedde S, et al. (2005) Thyroid hormone increases mannan-binding lectin levels. Eur J Endocrinol 153: 643-649.

39. Copeland JL, Verzosa ML (2014) Endocrine response to an ultra-marathon in pre- and post-menopausal women. Biol J Sport 1: 125-131. 